\title{
Rapid Photometric and Spectroscopic Variability of the Be Star DX Eri
}

S. Štefl

\author{
Astronomical Institute, Academy of Sciences, Ondřejov, Czech Republic \\ L. A. Balona \\ South African Astronomical Observatory, P.O. Box 9, Observatory \\ 7935, Cape, South Africa
}

\begin{abstract}
Short-period variations of high-resolution He I $667.8 \mathrm{~nm}$ line profiles obtained on 5 nights are analysed and compared to extensive uvby photometry obtained during $1986-1993$. The separation of shortperiod and medium-term light variations was done for 4 seasons. All prewhitened magnitudes can be phased with a single period of $1.2564 \mathrm{~d}$. The radial velocity and the first moment of the HeI 667.8 line follows the photometric period with a semi-amplitude of $42 \mathrm{~km} \mathrm{~s}^{-1}$. The large amplitude is very difficult to understand in terms of current models of non-radial pulsation or star spots.
\end{abstract}

\section{Introduction}

We analysed 280 new uvby observations of the Be star DX Eri (56 Eri=HR 1508) obtained at ESO, La Silla and SAAO during 1991 - 1993 together with those published by Balona et al. (1992). In addition, 28 high-resolution He I $667.8 \mathrm{~nm}$ profiles were obtained with the ESO 1.4-m telescope during 5 nights in November 1991. The resolving power is 70000 , temporal sampling $60-80$ minutes and typical $\mathrm{S} / \mathrm{N} \approx 200$.

The purpose is to derive the period of the rapid variations, to determine the colour variation and to model the relationship between the photometric and spectroscopic parameters by means of non-radial pulsations and star spots. In this way we hope to discriminate between the two models.

\section{Results}

Random medium-term (with a characteristic time scale of about 10 days) and short-period light variations combine in a complicated pattern. In order to separate the strictly periodic, variation characteristic of many Be stars (the $\lambda$ Eri phenomenon), it was necessary to employ a smoothing procedure.

\footnotetext{
${ }^{1}$ Based on data collected at the ESO and SAAO.
} 
Time series analysis of smoothed $b$-magnitudes in individual seasons gives significant periods in the interval $1.241-1.369 \mathrm{~d}$. We cannot determine whether the differences reflect intrinsic variations in the period or whether they are caused by imperfect prewhitening. Nevertheless, all smoothed $b$-magnitudes in the interval $1986-1993$ are well phased with one single period of $1.256 \mathrm{~d}$.

The colour-index variations corresponding to the periodic component were derived from the prewhitened uvby magnitudes obtained in 1991 and 1993. The following conclusions can be drawn regarding this component:

- All significant variations are in phase. The semi-amplitude of the brightness variation $\left(0 .^{m} 08-0 .^{m} 10\right)$ is comparable in all passbands and seasons.

- The light curves are not symmetrical: light maxima occur near phase $0 . P 60$.

- The $m_{1}$ index is constant within the errors, but the amplitude of $b-y$, $u-b$ and $c_{1}$ varies from season to season.

- The star is bluest when brightest.

The line-profiles show low-degree line-asymmetry variations. The radial velocity (RV) measured in the line wings follow the photometric $1.256 \mathrm{~d}$ period with a semi-amplitude of $41 \mathrm{~km} \mathrm{~s}^{-1}$. The RV minimum is shifted by $0 . P 25-0 . P 30$ with respect to light minimum. The first line moment follows the same curve with a lower amplitude and larger scatter.

The large radial velocity amplitude cannot be understood as a result of the pulsational displacement alone. Statistical results obtained by comparing the photometric periods and projected rotational velocities show that the period in the rotating frame is at least 4 times larger than the observed period (a three standard deviation result; Balona 1990). This, coupled with the large RV amplitude leads to an unrealistically high fractional radius variation. If pulsation is involved, its main effect must be a change in temperature due to compression and decompression.

The net result is that the NRP model is observationally similar to a large star spot which drifts slowly across the photosphere. However, a star spot model cannot account for the large amplitude either, since the expected semi-amplitude is only about $20 \mathrm{~km} \mathrm{~s}^{-1}$.

This study is a second attempt, after that of $\eta$ Cen (Štefl et al. 1995), to derive relations between the rapid photometric and spectroscopic variations. In $\eta$ Cen the medium-term variations occur on a considerably longer time-scale. An interesting difference between the two stars is that the minima of light and RV coincide for $\eta$ Cen, but are shifted by a quarter of the period in DX Eri.

\section{References}

Balona, L.A. 1990, MNRAS, 245, 92

Balona, L.A., Cuypers, J., \& Marang, F. 1992, A\&AS, 92, 533

Štefl, S., Baade, D., Harmanec, P., \& Balona, L.A. 1995, A\&A, in press 